\title{
Successfully Resected Cardiac Metastatic Melanoma in a Lifesaving Cardiac Surgery: A Case Report
}

\author{
Petar Vukicevic, MD, PhD, ${ }^{1,6}$ Milos Matkovic, MD, ${ }^{2,5}$ Ivan Markovic, MD, PhD, ${ }^{3,5}$ \\ Aleksandar Mikic, MD, PhD, ${ }^{2,5}$ Svetozar Putnik, MD, PhD, ${ }^{2,5}$ Vesna Bozic, MD, ${ }^{4}$ Nemanja Aleksic, $M D, 2,5$ \\ Vladimir Cvetic, MD, ${ }^{5,7}$ Vladimir Tutus, MD, ${ }^{2,5}$ Radan Dzodic, MD, PhD ${ }^{3,5}$ \\ ${ }^{1}$ Department for Cardiac Surgery, Military Medical Academy, Belgrade, Serbia; ${ }^{2}$ Department for Cardiac Surgery, Clinical Center of \\ Serbia, Belgrade, Serbia; ${ }^{3}$ Clinic of Surgical Oncology, Institute of Oncology and Radiology of Serbia, Belgrade, Serbia; ${ }^{4}$ Department for \\ Cardiovascular pathology, Clinical Center of Serbia, Belgrade, Serbia; ${ }^{5}$ University of Belgrade, Faculty of Medicine, Belgrade, Serbia; \\ ${ }^{6}$ University of Defense, Faculty of Medicine, Belgrade, Serbia; ${ }^{7}$ Department for Radiology, Clinical Center of Serbia, Belgrade, Serbia
}

\section{ABSTRACT}

Introduction: Metastatic tumors are the most frequent tumors of the heart with the melanoma metastasis being the most frequent. In cases of a cardiac tamponade or a low cardiac output syndrome due to the position of the tumor it could lead to an acute life-threatening condition for the patient.

Case report: We present a case of a successfully treated metastatic cardiac melanoma in a lifesaving cardiac surgery. A 42-yearold woman was admitted to the emergency department of the Clinical Center of Serbia in a critical state with signs of tricuspid orifice obstruction with a tumor mass. Her previous medical history showed that she had an adequate surgical excision of the melanoma in the right lumbar region at the age of 39. An emergency cardiac surgery was performed with the resection of the tumor and the atrial wall. The postoperative course was uneventful and a HP exam once again confirmed the same type of melanoma as previously diagnosed. The patient was alive and well on followup exams for 4 months when she was diagnosed with metastatic tumor masses in the pelvis with ascites and melanosis of the entire skin followed by lethal outcome 5 months after the cardiac surgery.

Conclusion: Surgical resection of metastatic cardiac melanoma can be safe and effective in an emergency scenario, especially in the case of solitary metastasis. It can be performed with excellent results and very few postoperative complications. However, due to the nature of the principal disease, the long-term survival rate remains low, giving the surgery a place in palliative treatment.

\section{INTRODUCTION}

Cardiac melanoma can be found in up to $65 \%$ of autopsies of patients with melanoma. [Glancy 1968; Aerts 2014] As

Received May 27, 2020; received in revised form fune 9, 2020; accepted fune 9, 2020.

Correspondence: Petar Vukicevic, Department for Cardiac Surgery, Military Medical Academy, Crnotravska 17, 11040 Belgrade, Serbia +38163455383 (e-mail: petar: vukicevic@gmail.com). patients with cardiac melanoma rarely express symptoms, they are rarely diagnosed ante mortem. Clinical symptoms could be non-specific, such as fatigue or general weakness. Depending on cardiac location, symptoms vary from pericardial effusion to congestive heart failure [Ozyuncu 2006]. As melanoma with cardiac metastasis occurs in advanced stages of the disease, it carries a poor prognosis; thus surgical treatment is rarely considered and is mostly palliative. We present a case of a successfully resected cardiac melanoma in a lifesaving cardiac surgery.

\section{CASE REPORT}

A 42-year-old woman was admitted to the emergency department of the Clinical Center of Serbia in a critical state, hypotensive, heavily dyspneic, tachycardic with pretibial edema, and anuric. On admission, arterial tension was 85/60 $\mathrm{mmHg}$ with a heart rate of 125 beats per minute and oxygen saturation of $80 \%$ on nasal oxygen. After placement of the central venous line, the central venous pressure was measured at $18 \mathrm{mmHg}$. An orientational echocardiography was immediately performed, revealing masses in the right heart cavities with near total obstruction of tricuspid valve and filiform blood flow through the tricuspid orifice. The patient was intubated and rushed into the CT scan, which confirmed a tumor mass in the right ventricle and right atrium (Figure 1). The patient was put on inotropes and transferred to the cardiac surgery department to undergo urgent surgery.

Due to well-documented medical history and data given by accompanying relatives, we learned that the patient had an adequate surgical excision of the melanoma in the right lumbar region at age 39. The histopathology exam revealed invasive skin melanoma (CLARK II, BRESLOW III- 8.75 $\mathrm{mm}$, nodular type, with presence of mitosis, superficial ulceration less than $3 \mathrm{~mm}$ and lymphocytic response). After the complete radical excision, the patient was included in AJCC, EORTC trial 18961 and given postoperative adjuvant Ganglioside GM2-KLH/QS-21 vaccination treatment [Eggermont 2013]. She received eleven doses of the vaccine and was without signs of primary tumor or metastasis on regular follow-up exams for two years. After two years, a pigment 

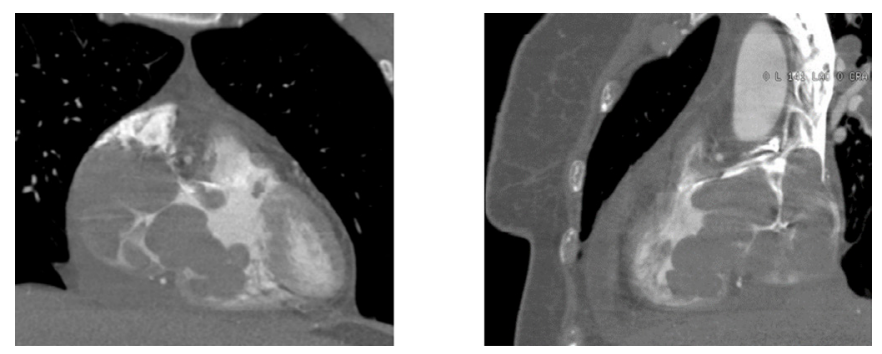

Figure 1. CT scan findings of tumor mass in the right ventricle and right atrium.
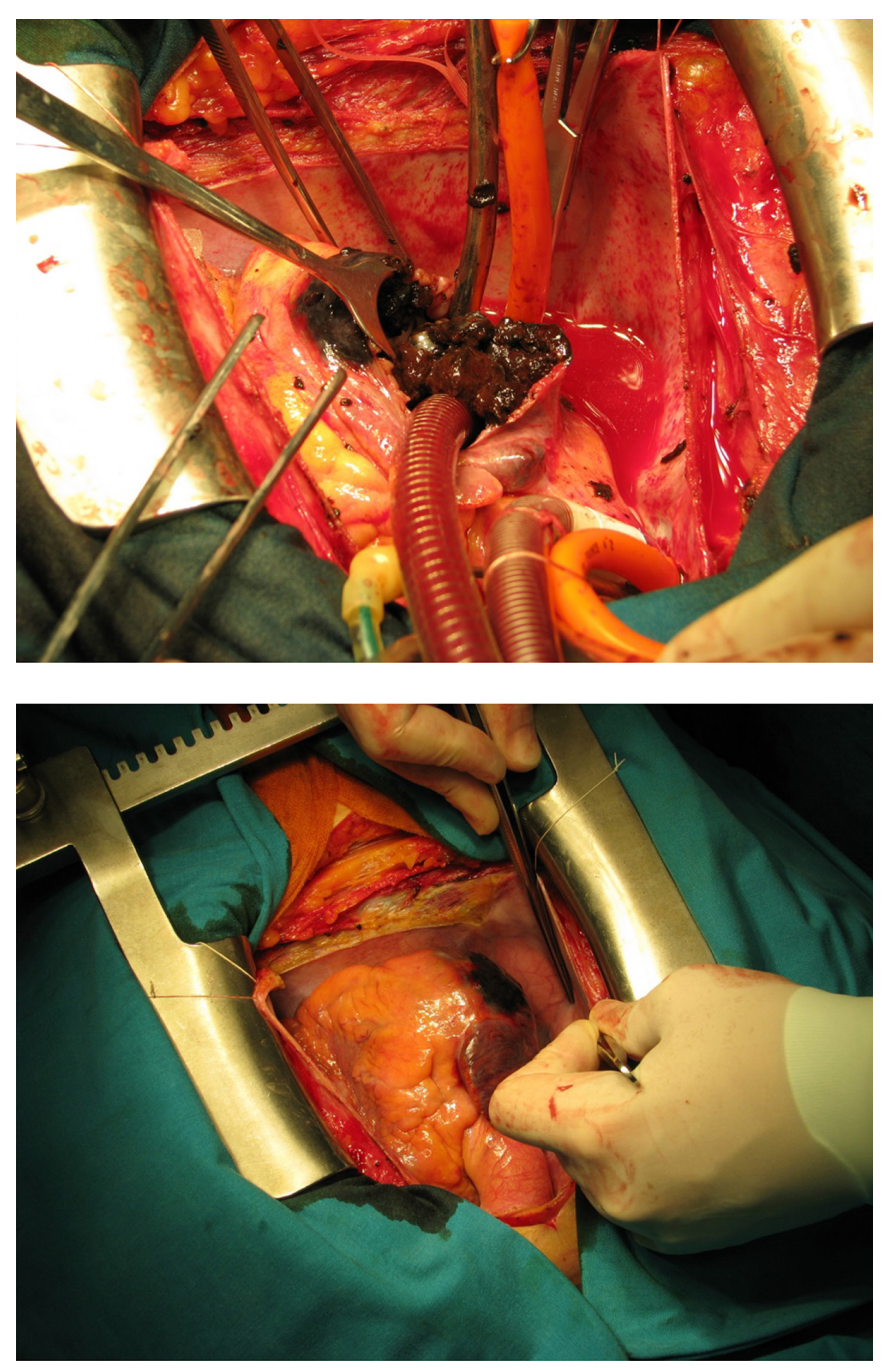

Figure 2. Intraoperative finding of cardiac melanoma in the right heart cavities.

tumor mass in the projection of 12 th rib in right hemithorax was found. Radical excision was performed. The HP findings were again those of invasive melanoma with CLARK III and BRESLOW III stages. The following control exams showed no local relapses, absence of peripheral lymphadenopathy, or signs of metastatic disease on abdominal echosonography

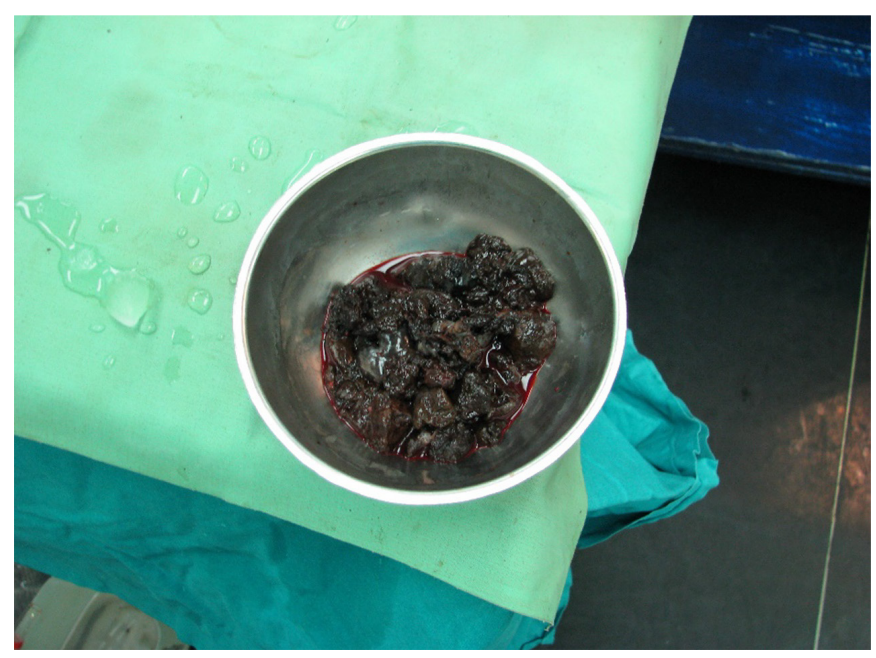

Figure 3. Removed friable tumor mass.

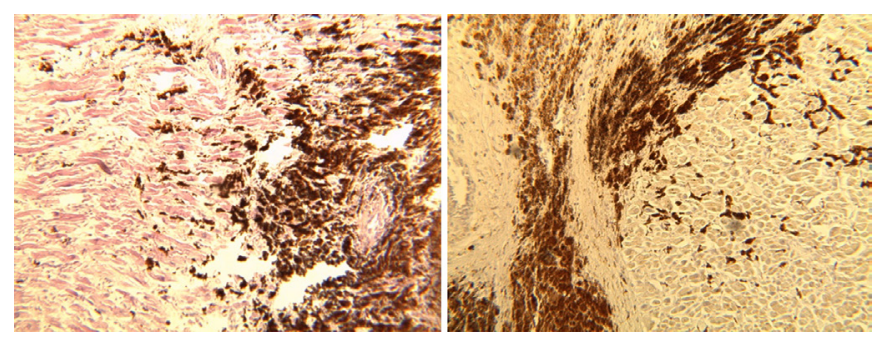

Figure 4. Histopathological findings of the cardiac melanoma.

and abdominal CT scans. Until admission to the emergency department, the echocardiography had not been performed on follow-up exams.

The urgent cardiac surgery was performed immediately after the CT scan. The patient was transferred to the operating room, unstable, and on inotropic support. Median sternotomy was performed and the patient was put on cardiopulmonary bypass. The heart was arrested using cold crystalloid St. Thomas cardioplegic solution. The intraoperative findings revealed a large dark grey tumor mass, which infiltrated the right atrium free wall extending through the tricuspid valve into the right ventricle with near complete obstruction of the orifice (Figure 2). The friable tumor mass (Figure 3) was completely removed through the right atriotomy. The infiltrated part of the right atrium wall was completely resected. Detailed lavage of the right cardiac cavities and pericardium was performed and the right atrium wall was closed via direct suture. There was no involvement of the valve leaflets or subvalvular apparatus. The total size of the excised tumor was $60 \times 50 \times 30$ $\mathrm{mm}$ weighing approximately 40 grams. The postoperative course was uneventful and an HP exam once again confirmed the same type of melanoma as previously diagnosed (Figure 4). Also, the HP exam showed that $95 \%$ of the tumor mass was necrotic. The control echocardiography showed no residue of tumor masses in the heart cavities. The patient was discharged on day 7. The multidisciplinary melanoma team advised postoperative chemotherapy, which the patient refused. 
The patient was alive and well on follow-up exams for 4 months, when she was diagnosed with metastatic tumor masses in the pelvis with ascites and melanosis of the entire skin, followed by lethal outcome 5 months after the cardiac surgery.

\section{DISCUSSION}

Metastatic tumors are the most frequent tumors of the heart, with melanoma metastasis being the most frequent. In the case of a cardiac tamponade or a low cardiac output syndrome due to the position of the tumor, it could lead to an acute life-threatening condition for the patient [Judge 2013].

The preferred diagnostic tool for right-sided lesions is the transesophageal echocardiography [Obeid 1993]. In the case of our patient, the echocardiography had not been performed up until admission to the emergency room. Cardiac MRI is a valuable resource in the case of this tumor, giving the specific signal hyperintensity pattern on $\mathrm{T} 1$ and $\mathrm{T} 2$ sequences. The gadolinium enhancement is present in nearly all cases seen in MRI exams of these patients. The F-FDG PET/CT scan is useful and accurate for the lesions less than $1 \mathrm{~cm}$ in diameter [Mousseaux 1998]. An endovascular cardiac-biopsy specimen may show intramural cardiac metastatic melanoma, with tumor cells that were positive for a panel of melanocytic markers, including SOX10 marker antibodies [Safa 2019]. In the case of our patient, the CT scan was chosen instead because of the condition of the patient and shorter exam time than in an MRI and endovascular cardiac-biopsy.

The five-year survival for stage III and IV melanoma is up to $20 \%$ and depends on systemic therapy, surgical resection, and comorbidities. The median of survival for patients with metastatic melanoma is 6.4 months. For the patients with cardiac involvement, 6-month survival is low, ranging up to $23 \%$ [Manola 2000]. Having in mind these results, the surgical treatment for cardiac melanoma in literature has been mostly palliative. The successful resections from both atria and ventricles have been described and several groups report an improvement in quality of life after one year [Gibbs 1999; Houmsse 2004; Onan 2010; Kontozis 2011]. The resection techniques depend on the local extension of the tumor and the heart structures involved. The aim is complete resection if possible, with the patch or direct closure of the cavities if involved by tumor. Nevertheless, in some cases of metastatic cardiac melanoma with extensive cardiac involvement, complete and safe resection of the tumor is not possible and the tumor should be excised as extensively as possible [Morosin 2019]. In our case, the extremely friable tumor was completely removed. The right atria wall that was resected was closed with direct suture, and there was no need for a patch due to the size of the defect. Operative techniques range even up to ex-vivo resection and auto transplantation [Judge 2013].

The medical treatment following cardiac surgery should be guided by the oncology team. Until 2010, the only FDAapproved drug for this indication was Dacarbazine, with response rate of $15 \%$ and long-term complete response of only 1-2\% [Middleton 2000]. The high dose IL-2 immunotherapy offered another option, with a long-term response of up to $6 \%$. However, both options showed significant toxicity. After 2011, the FDA approved two new drugs: ipilimumab and vemurafenib. Vemurafenib is a selective inhibitor of a form of constitutively activated mutant BRAF serinethreonine kinase present in approximately $50 \%$ of melanoma. It showed superior effect to dacarbazine with a progressionfree survival of 5.3 months [Middleton 2000]. Thus, BRAF mutation presence testing could be justified in a stable and non-hemodynamically compromised patient. Ipilimumab is an anti-CTLA-4 receptor antibody, which aims to block inhibitory downregulatory signals to T-cells, thus promoting T-cell survival and activation. Its response rate is slower but more durable, as the trials have showed. It can be used as complementary therapy to vemurafenib [Judge 2013].

The data on external beam radiation in literature are scattered, and one report suggests that it may have some beneficial effect on cardiac melanoma [Magnuson 2010]. The patient in our case refused any suggested systemic hemotherapy after the surgery.

Having all this data in mind, the assessment of each patient on a case by case basis should be the preferred approach. In a stable patient with cardiac involvement, there should be a multidisciplinary approach, led by the oncology team who will most suitably decide on the recommended therapy based on the type of tumor and patient characteristics. For an asymptomatic patient with solitary cardiac metastasis, palliative surgery with curative intent with clear margin resection, if possible, is recommended. In case of an unstable patient, emergency surgery is recommended, with partial or full resection, if applicable, as a lifesaving procedure.

Also, the routine echocardiography during follow up of the previously treated melanoma patient could be useful in early detection of metastasis before symptoms occur and the patient becomes unstable.

\section{CONCLUSION}

Surgical resection of metastatic cardiac melanoma can be safe and effective in an emergency scenario, especially in the case of solitary metastasis. Considering a poor preoperative condition, this resection can be performed with excellent results and very good postoperative clinical findings. However, due to the nature of the principal disease, the long-term survival rate remains low, giving the surgery a place in palliative treatment.

\section{REFERENCES}

Aerts BRJ, Kock MCJM, Kofflard MJM, Plaisier PW. 2014. Cardiac metastasis of malignant melanoma: a case report. Neth Heart J 22:39-41.

Eggermont AM, Suciu S, Rutkowski P, et al. 2013. Adjuvant ganglioside GM2-KLH/QS-21 vaccination versus observation after resection of primary tumor $>1.5 \mathrm{~mm}$ in patients with stage II melanoma: results of the EORTC 18961 randomized phase III trial. J Clin Oncol 31:3831-7.

Gibbs P, Cebon JS, Calafiore P, Robinson WA. 1999. Cardiac metastases from malignant melanoma. Cancer 85:78-84. 
Glancy DL, Roberts WC. 1968. The heart in malignant melanoma. A study of 70 autopsy cases. Am J Cardiol 21:555-71.

Houmsse M, Raman SV, Leier CV, Orsinelli DA. 2004. Metastatic melanoma of the left ventricle: cardiac imaging in the diagnosis and surgical approach. Int J Cardiovasc Imaging 20:523-6; discussion 527-8.

Judge JM, Tillou JD, Slingluff Jr CL, Kern JA, Kron IL, Weiss GR. 2013. Surgical management of the patient with metastatic melanoma to the heart. J Card Surg 28:124-8.

Kontozis L, Soteriou M, Papamichael D, et al. 2011. Isolated right atrial metastasis of malignant melanoma mimicking a myxoma. Hellenic J Cardiol 52:281-4.

Magnuson WJ, Halligan JB. 2010. Successful treatment of melanoma metastatic to the left atrium using external beam radiation therapy. Oncology (Williston Park) 24:650-3.

Manola J, Atkins M, Ibrahim J, Kirkwood J. 2000. Prognostic factors in metastatic melanoma: a pooled analysis of Eastern Cooperative Oncology Group trials. J Clin Oncol 18:3782-93.

Middleton MR, Grob JJ, Aaronson N, et al. 2000. Randomized phase III study of temozolomide versus dacarbazine in the treatment of patients with advanced metastatic malignant melanoma. J Clin Oncol 18:158-66.

Mousseaux E, Meunier P, Azancott S, Dubayle P, Gaux JC. 1998. Cardiac metastatic melanoma investigated by magnetic resonance imaging. Magn Reson Imaging 16:91-5.

Morosin M, Azzu A, Capoccia M, Rosendahl U. 2019. Extensive cardiac infiltrative melanoma. J Card Surg 34:858-9.

Obeid AI, al Mudamgha A, Smulyan H. 1993. Diagnosis of right atrial mass lesions by transesophageal and transthoracic echocardiography. Chest 103:1447-51.

Onan B, Onan IS, Polat B. 2010. Surgical resection of solitary metastasis of malignant melanoma to the right atrium. Tex Heart Inst J 37:598-601.

Ozyuncu N, Sahin M, Altin T, Karaoguz R, Guldal M, Akyurek O. 2006. Cardiac metastasis of malignant melanoma: a rare cause of complete atrioventricular block. Europace 8:545-8.

Safa H, Glitza Oliva IC. 2019. Cardiac metastases in melanoma. N Engl J Med 380:858. 